Published in final edited form as:

Ann Biomed Eng. 2008 April ; 36(4): 554-562. doi:10.1007/s10439-007-9426-3.

\title{
Effects of Disturbed Flow on Endothelial Cells
}

\author{
Shu Chien \\ Departments of Bioengineering \& Medicine, and Whitaker Institute for Biomedical Engineering, \\ University of California, San Diego, PFBH room 134, 9500 Gilman Drive, La Jolla, CA \\ 92093-0412, USA
}

\begin{abstract}
Vascular endothelial cells (ECs) play significant roles in regulating circulatory functions. The shear stress resulting from blood flow modulates EC functions by activating mechano-sensors, signaling pathways, and gene and protein expressions. Shear stress with a clear direction resulting form pulsatile or steady flow causes only transient activation of pro-inflammatory and proliferative pathways, which become down-regulated when such directed shearing is sustained. In contrast, shear flow without a definitive direction (e.g., disturbed flow in regions of complex geometry) causes sustained molecular signaling of pro-inflammatory and proliferative pathways. The EC responses to shear flows with a clear direction involve the remodeling of EC structure to maintain vascular homeostasis and are atheroprotective. Such regulatory mechanism does not operate effectively when the flow pattern is disturbed. Therefore, the branch points and other regions of the arterial tree with a complex geometry are prone to atherogenesis, whereas the straight part of the arterial tree is generally spared. Understanding of the EC responses to different flow patters helps to elucidate the mechanism of the region-specific localization of atherosclerosis in the arterial system.
\end{abstract}

\section{Keywords}

Gene expression; Mechanotransduction; Proliferation; Shear stress; Signal transduction

\section{INTRODUCTION}

Endothelial cells (ECs) perform many important functions such as the regulation of permeability, migration, remodeling, proliferation, and apoptosis, the production, secretion, and metabolism of biochemical substances, as well as the modulation of contractility of vascular smooth muscle cells (SMCs). ECs respond to mechanical factors, e.g., fluid shear stress, which can also be sensed by the EC to modify intracellular signaling, gene expression, protein expression, and cell functions. 4

The fluid shear stress that acts parallel to the cell surface is a product of fluid viscosity and the velocity gradient between adjacent layers of the flowing fluid. The pioneering work by Goldsmith and his colleagues provided the most definitive characterization of the variations in flow patterns in the vascular system10,13,16,17 showing that the marked variations in flow streams and shear stress in regions of curvature, branching, and other complex geometries. Subsequent investigations have found that the EC structure and function differ

\footnotetext{
(C) 2007 Biomedical Engineering Society

Address correspondence to Shu Chien, Departments of Bioengineering \& Medicine, and Whitaker Institute for Biomedical Engineering, University of California, San Diego, PFBH room 134, 9500 Gilman Drive, La Jolla, CA 92093-0412, USA. Electronic mail: shuchien@ucsd.edu.
} 
significantly between the straight part of the arterial tree, where the flow has relatively straight streamlines with well-defined directions, as compared to branch points and curved regions that have complex flow patterns (e.g., 2,8,28). This presentation summarizes the work done in our laboratory on mechanotransduction in the EC in response to these different patterns of shear stress. These results, together with the data in the literature, have led to the formulation of the concept that the variations in shear stress patterns can result in different consequences of mechanotransduction, leading to the predilection for atherosclerosis at branch points and curved regions and the protection against atherogenesis in the straight part of the arterial tree.

\section{IN VITRO STUDIES ON ENDOTHELIAL RESPONSES TO DIFFERENT PATTERNS OF SHEAR STRESS}

Mechanotransduction in ECs has been studied by using both in vitro and in vivo approaches. The advantages of the in vitro studies are that the experimental variables, including the mechanical conditions, can be well controlled. In vivo studies are important for assessing the applicability and relevance of the in vitro findings to physiological and pathophysiological conditions.

Flow chambers have been used for in-vitro studies on the effects of shear stress on cultured ECs. The parallel-plate rectangular flow chamber with a uniform channel height along the flow path can be used to study the effects of steady or pulsatile laminar shear flow (Fig. 1a), as well as reciprocating shear flow on a monolayer of cultured ECs. The step flow chamber6 with a vertical step expansion of channel height at the entrance can be used to study the effects of disturbed shear (Fig. 1b). The flow patterns beyond the step consist of a region with flow recirculation in a direction opposite to the inflow, a region of flow reattachment (where wall shear stress is near zero, but shear stress gradient is high), and the transition to a region of forward flow similar to that seen in the flow chamber with a uniform height. Thus, the step flow chamber allows the study of the responses of ECs to disturbed flow in the vicinity of the reattachment area and laminar flow downstream in the same chamber.

\section{Effects of Shear Stress on EC Mechanosensing and Intracellular Signaling In Vitro}

The application of shear stress to ECs can activate a number of mechano-sensors. 3 These include membrane proteins such as the vascular endothelial growth factor receptor Flk-1 (a receptor tyrosine kinase), the integrins (e.g., $\alpha_{\mathrm{v}} \beta_{3}$ ), G-proteins and G-protein coupled receptors, ion channels, and intercellular junction proteins, as well as membrane lipids and glycocalyx.

The shear stimulation of the sensors, acting through adaptor molecules (e.g., Shc, Grb2, and Sos), triggers a cascade of signaling pathways, and consequently modulates the expression of genes and proteins relevant to EC functions in health and disease.20 The mechanotransduction processes depend on the mode of shearing. In general, shear stress with a significant forward direction (whether steady shear stress without oscillation or pulsatile shear stress in which an oscillatory component is superimposed on steady shearing) generate comparable effects. In contrast, shearing without a significant forward direction, e.g., reciprocating flow with little net forward component or disturbed flow generated near the reattachment zone in a step flow chamber (Fig. 1b), yield results that tend to be opposite to shearing with a significant forward direction.

\section{Effects of Shear Stress on EC Expression of Monocyte Chemotactic Protein-1}

The expression of the MCP-1 gene is modulated by the Ras-MAPK (mitogen activated protein kinases) pathway. The application of shear stress with a significant forward direction 
(e.g., 1.2 Pa) to ECs causes Ras to bind GTP, and the sequential activation of MAPKs and MCP-1 expressions, 30 but these responses are transient in nature in that sustained shearing causes the deactivation/downregulation of the Ras-MAPK system and the expressions of MCP-1 and various pro-inflammatory or pro-proliferative molecules to below the static control levels.21,30 In regions with disturbed flow, the activation of the MAPK pathway is sustained, and so are the downstream gene and protein expressions. The functional consequence of the down-regulation of MCP-1 by sustained shearing with a forward direction is a suppression of monocyte attraction into the vessel wall and is thus atheroprotective. In concert with this concept, it has been found that the induction of MCP-1 expression by oxidative stress is attenuated by pulsatile shear stress with a forward direction and augmented by reciprocating shear stress without a forward direction.14

\section{Effects of Shear Stress on EC KLF-2 Expression and Survival}

Krüppel-like factor-2 (KLF-2), a member of the KLF family zinc finger-containing transcription factors, is abundantly expressed in ECs18 and is beneficial to EC survival, e.g., in response to oxidized LDL.36 We have contrasted the effects of pulsatile shear stress (1.2 $\pm 0.4 \mathrm{~Pa}$ at $1 \mathrm{~Hz}$ ), which has a significant forward direction (Fig. 2a), with those of reciprocating shear stress $(0.05 \pm 0.4 \mathrm{~Pa}$ at $1 \mathrm{~Hz})$, which has a minimal forward direction (Fig. 2b), on the expression of KLF-2 in HUVECs.36 The KLF2 mRNA level increases significantly after exposure to both pulsatile and reciprocating shear stresses at $1 \mathrm{~h}$ (Figs. 2c and $2 \mathrm{~d}$ ). The continued application of pulsatile shear stress leads to a sustained up-regulation of KLF2 for as long as $24 \mathrm{~h}$ (Fig. 2c). In contrast, the continued application of reciprocating shearing causes the KLF2 gene expression to decrease to below the basal level at $4 \mathrm{~h}$ and remains low throughout the $24 \mathrm{~h}$ of shearing (Fig. 2d). These results indicate that 24-h reciprocating shear stress, which lacks a significant forward component, inhibits KLF2 expression and decreases the ability of ECs to survive against atherogenic stimuli such as oxidative stress. 24-h pulsatile shear stress, which has a significant forward component, results in continued expression of KLF2 and hence is beneficial to EC survival.

\section{Effects of Shear Stress on EC Proliferation}

DNA microarray studies on the effects of steady laminar shear stress at $1.2 \mathrm{~Pa}$ for $24 \mathrm{~h} 40$ on cultured HAECs showed that several genes related to EC proliferation and inflammation are down-regulated, whereas a number of genes involved in EC survival and angiogenesis (e.g., Tie2 and Flk-1) and vascular remodeling (e.g., matrix metalloproteinase-1) are up-regulated, suggesting that sustained shearing with a forward component keeps the EC gene expression profile in a non-proliferative and non-inflammatory state. In concert with these results on gene expression, we have found that such shearing increases the expression of the growth arrest proteins GADD45, $\mathrm{p} 21^{\mathrm{cip}} 1$, and $\mathrm{p} 53$, and decreases the phosphorylation of the retinoblastoma gene product $(\mathrm{Rb})$, thus leading to cell cycle arrest.22

In contrast to the anti-proliferative actions of laminar shear stress with a forward component, studies in the step flow chamber show that the incorporation of BrdU into cultured ECs is enhanced in the region of disturbed flow in the re-attachment area and its vicinity, while that BrdU incorporation is much lower in the downstream laminar flow region.2 The same distribution pattern is seen for the activation of signaling molecules for proliferation such as ERK. The increase in BrdU incorporation induced in the area of disturbed flow can be blocked by the ERK inhibitor PD98059. These results indicate that the flow pattern in the re-attachment area at branch points stimulates cell proliferation via ERK activation, and this is to be contrasted with the low cell proliferation rate due to the up-regulation of growtharrest genes when ECs are exposed to shearing with a significant forward component. 


\section{Effects of Shear Stress on Intercellular Junctions}

Shear stress can modulate the intercellular junction proteins such as connexins 19 and PECAM-1.34 VE-cadherin is an intercellular adhesion molecule that plays a role in mediating endothelial integrity and permeability. VE-cadherin staining of the ECs in the step flow chamber showed that prolonged exposure of ECs to pulsatile flow $(1.2 \pm 0.4 \mathrm{~Pa}$ at 1 $\mathrm{Hz}$ ) results in a continuous staining of VE-cadherin at cell borders in the laminar flow region, but discontinuous staining in the disturbed flow region (Fig. 3). The application of reciprocating flow $(0.05 \pm 0.4 \mathrm{~Pa}$ at $1 \mathrm{~Hz})$ in a rectangular flow chamber results in a discontinuous staining similar to that seen in the disturbed flow region.27 The ECs in regions of disturbed flow in the step flow chamber also show a loss of peripheral F-actin microfilaments in the periphery. 6 These redistributions of gap junctions and cytoskeletal proteins in regions of flow disturbance may contribute to intercellular widening, and consequently an increase in macromolecular permeability.

\section{Effects of Shear Stress on EC Lipid Metabolism}

We have investigated the effects of different flow patterns on the EC activity of sterol regulatory element binding protein 1 (SREBP1), which can increase the expressions of genes encoding for LDL receptor, cholesterol synthase, and fatty acid synthase, thus increasing the intracellular sterol level. The application of laminar shear stress (1.2 Pa) causes a transient activation of SREBP1 and the ensuing translocation of its transcription factor domain into the nucleus.24 Studies using the step flow chamber indicate that, in contrast to the transient activation of SREBP1 in ECs under steady flow, disturbed flow causes a sustained activation of SREBP1 (Fig. 4), which would lead to transcriptional activation of EC genes encoding for the genes that tend to impair lipid homeostasis in ECs.

\section{Effects of Shear Stress on Cytoskeleton Organization and Cell Morphology}

In ECs exposed to sustained shear stress with a clear direction, staining of cytoskeleton proteins (actin, tubulin, and vimentin) shows that the cytoskeletal fibers undergo remodeling to become oriented with the direction of shear flow, 11 with a consequent alignment of the cell with the shear flow direction. Such cytoskeletal remodeling is not seen under disturbed flow, with the cytoskeletal fibers and the cell showing random orientation similar to that seen in static condition. BAECs subjected to oscillatory shear stress without a net direction also have a morphology and random orientation similar to that of static controls. 32

\section{IN VIVO STUDIES ON ENDOTHELIAL RESPONSES TO DIFFERENT PATTERNS OF SHEAR STRESS}

In vivo, the flow in the straight part of the arterial tree is pulsatile with a marked forward flow, whereas that at the branch points has a complex pattern with little forward component16,17 and is similar to the reciprocating shearing in the rectangular flow chamber and in the reattachment zone in the step flow chamber.6

\section{Effects of Local Shear Stress Pattern on KLF2 and MCP-1 Expressions in ECs In Vivo}

We have examined the branch areas between the abdominal aorta and the celiac artery of normal rats by immunostaining with an anti-KLF2 antibody and histological sections, with the goal of determining whether the flow-pattern-specific regulation of KLF2 expression found in cultured ECs in vitro also exists in the native circulation in vivo. 36 In the abdominal aorta, KLF2 expression is continuous throughout the luminal lining (Fig. 5, Region 3). In the celiac artery, marked differences in KLF2 expression are observed between the medial and lateral aspects. The expression level of KLF2 on the medial flow divider apex (Fig. 5, Region 2), where the shear stress is high and laminar, is considerably 
higher than that of the lateral aspects (Fig. 5, Region 1), where disturbed flow is more prone to occur.

To further assess the effect of a change in flow pattern from forward to disturbed flow on KLF2 expression in vivo, we used a stenosis model in which the rat abdominal aorta was subjected to partial constriction (Fig. 6a). Examination of the aorta by immunohistochemistry 4 weeks later shows high KLF2 expression in ECs at regions of pulsatile flow with a clear forward direction (Fig. 6b), but virtually no expression in ECs at the poststenotic site, where the flow pattern is disturbed (Fig. 6c).36 These results, together with our observation in rat artery bifurcation (Fig. 5), indicate that the modulation of KLF2 expression by different flow patterns in vivo is similar to that observed in vitro (Fig. 2).

MCP1 protein expression examined by immunocytochemistry has shown a preferential expression near the intercostal artery orifices in comparison to the straight part of the aorta2; mapping of monocyte distribution in the aorta also shows that there is a preferential localization at branch points. 25 The expression patterns of KLF2 and MCP1 at branch points vs. straight vessel in vivo are similar to what was observed in response to reciprocating/disturbed flow vs. pulsatile or steady laminar flow in vitro. These in vivo findings suggest that local flow patterns can affect EC functions through differential regulations of the atherogenic vs. atheroprotective gene products.

\section{Effects of Local Shear Stress Pattern on EC Turnover, Junction Proteins, and Macromolecular Permeability In Vivo}

At the branch points and curved regions of the arterial tree, which have a predilection for atherosclerosis, blood flow is unsteady and the shear stress shows marked spatial and temporal variations. 12 Our theoretical and experimental studies have provided evidence that EC turnover, including mitosis and death,5,37 are associated with an increase in permeability of macromolecules (e.g., LDL, albumin, and the electron-dense marker horseradish peroxidase) across the leaking intercellular junctions.1,15 Studies performed in a number of laboratories, including our own, have shown that these events of accelerated EC turnover occur primarily in areas with disturbed blood flow, e.g., arterial branch points. $7,23,33$

The potential role of hemodynamic factors and EC turnover in the focal nature of lipid infiltration has been studied in rabbit thoracic aorta. 2 The distribution of shear flow patterns has been inferred from the nuclear orientation and shape index, and the results suggest that flow pattern is rather complex near the small branches, similar to that reported by Karino and Goldsmith.16,17 Mitotic cells and macromolecular leaky spots are distributed primarily around the branch orifices, and their distribution patterns are similar to that of lipid accumulation in cholesterol-fed rabbits 29 and in human atherosclerotic lesions. 31 These results indicate that the complex flow patterns with little forward component enhance EC turnover and macromolecular permeability to lead to focal lipid accumulation.

Different flow patterns and associated shear stress have also been shown to regulate the expression and distribution of intercellular junctional proteins in ECs. Dai et al. 9 showed that the $\mathrm{Cx} 43$ expression is up-regulated by athero-prone waveform (mainly disturbed flow) of shear stress, whereas elevated Cx37 and Cx40 expression levels are associated with the atheroprotective waveform (mainly laminar flow). These observations in Cx patterns induced by athero-prone and athero-protective waveforms of shear stress are in agreement with the in vivo findings by Kwak et al., 19 who showed that $\mathrm{Cx} 43$ is detectable in the endothelium covering the shoulder of the plaques in human carotid arteries, whereas $\mathrm{Cx} 37$ and $\mathrm{Cx} 40$ are present in the endothelium of non-diseased arteries and not detectable in the endothelium covering the plaques. These results suggest a prominent role for complex 
hemodynamic flow conditions in determining regional differences in gap junction expression and cell-cell communication that may contribute to vascular pathological changes in regions of flow disturbance.

The expression of VE-cadherin at intercellular junctions is weak and discontinuous in the aortic arch (Figs. 7c and 7d), where the flow is disturbed, but significantly higher and continuous in the descending thoracic aorta and abdominal aorta (Figs. 7a and 7b), where flow is pulsatile and laminar with a forward component. 27 We used the model of partial constriction of the rat abdominal aorta described above (Fig. 6a) to examine the effect on VE-cadherin expression. Immunohistochemistry shows that VE-cadherin is highly expressed at EC borders in the abdominal aorta at sites where the blood flow is laminar (Fig. 6d). In contrast, there is little or no VE-cadherin staining in the EC borders in the poststenotic dilatation sites (Fig. 6e), where the flow is disturbed. These results, together with those shown in Fig. 5, indicate that different flow patterns modulate the VE-cadherin at EC junctions in vivo, similar to that observed in vitro (Fig. 2).

\section{Effects of Shear Stress on Cytoskeleton Organization In Vivo}

In the straight part of the aorta, EC stress fibers have prominent orientation parallel to the direction of blood flow.38,39 In contrast, there is little orientation of ECs or their stress fibers at branch sites lateral to the flow dividers, where flow patterns are more disturbed with no clear forward direction. It has been suggested that the prominent stress fibers with clear orientation at regions exposed to high levels of directional flows may help the ECs to withstand hemodynamic stress and maintain vascular integrity.26,39

Partial obstruction of arteries causes ECs in regions immediately downstream of the narrowing, where shear stress is low and unsteady, to become polygonal in shape with a loss of stress fiber orientation, in contrast to the EC elongation and stress fiber alignment with flow in regions of normal or high shear stress. 35 An increase in shear stress in canine common carotid artery following the creation of an arteriovenous shunt with the external jugular vein causes an increase in EC stress fibers. 26 These results indicate that the in vitro finding of orientation of stress fibers parallel to directional shear stress also occurs in vivo.

\section{SUMMARY AND CONCLUSIONS}

Vascular ECs, being situated at the interface between the flowing blood and the vessel wall, are directly exposed to changes in blood flow and the associated shear stress. Under normal conditions, ECs adapt to hemodynamic changes and optimize its functional performance.4 EC dysfunction, especially in regions of the arterial tree exposed to disturbed flow patterns, is a critical early event in atherosclerosis.9,12,28 In vitro and in vivo studies have shown contrasting effects between laminar shear flow with a definite direction (seen in the straight part of the arterial tree) and disturbed shear flow without a clear direction (seen at arterial branch points and curved regions) in terms of their signal transduction, gene expression, structure, and functions.4 It is generally agreed that the features in EC signaling and the functional consequences of disturbed flow play an important role in the initiation and progression of atherosclerotic lesions at regions of prevalence, The various systemic risk factors (e.g., hyperlipidemia, hypertension, smoking, obesity, and diabetes) superimpose on the effects of disturbed flow to cause the high propensity of atherogenesis in branch points and regions of curvature. In the relatively straight segments of an artery with a laminar flow and a physiological level of mean shear stress (1-7 Pa), ECs are aligned and elongated in the direction of flow.11 In contrast, in areas of disturbed flow, the ECs are more polygonal in appearance without a clear orientation, and there is a lack of organization of the cytoskeleton and intercellular junctional proteins (e.g., connexins9,19 and VE-cadherin27). These morphological changes are accompanied by increases in cell turnover rate and DNA 
synthesis and a sustained activation of SREBP, 24 which may consequently contribute to the increases in permeability to macromolecules such as lipoproteins, as well as lipid uptake and synthesis at the branch points. These differences in the effects of laminar flow with a defined direction and disturbed flow without a clear direction are summarized in Table 1.

\section{Acknowledgments}

This work was supported in part by grants HL43026, HL80518, and HL85159 from the National Heart, Lung, and Blood Institute. The author would like to acknowledge the valuable discussions with Dr. Harry Goldsmith and his inspirations on many of the experiments on blood cell rheology and flow dynamics.

\section{REFERENCES}

1. Chen YL, Jan KM, Lin HS, Chien S. Ultrastructural studies on macromolecular permeability in relation to endothelial cell turnover. Atherosclerosis. 1995; 118:89-104. [PubMed: 8579635]

2. Chien S. Molecular and mechanical bases of focal lipid accumulation in arterial wall. Prog. Biophys. Mol. Biol. 2003; 83:131-151. [PubMed: 12865076]

3. Chien S. Molecular basis of rheological modulation of endothelial functions: importance of stress direction. Biorheology. 2006; 43:95-116. [PubMed: 16687781]

4. Chien S. Mechanotransduction and endothelial cell homeostasis: the wisdom of the cell. Am. J. Physiol. Heart Circ. Physiol. 2007; 292:H1209-H1224. [PubMed: 17098825]

5. Chien S, Lin SJ, Weinbaum S, Lee MM, Jan KM. The role of arterial endothelial cell mitosis in macromolecular permeability. Adv. Exp. Med. Biol. 1988; 242:59-73. [PubMed: 3245515]

6. Chiu JJ, Wang DL, Chien S, Skalak R, Usami S. Effects of disturbed flow on endothelial cells. J. Biomech. Eng. 1998; 120:2-8. [PubMed: 9675673]

7. Chuang PT, Cheng HJ, Lin SJ, Jan KM, Lee MM, Chien S. Macromolecular transport across arterial and venous endothelium in rats. Studies with Evans blue-albumin and horseradish peroxidase. Arteriosclerosis. 1990; 10:188-197. [PubMed: 2180395]

8. Colangelo S, Langille BL, Gotlieb AI. Three patterns of distribution characterize the organization of endothelial microfilaments at aortic flow dividers. Cell Tissue Res. 1994; 278:235-242. [PubMed: 8001079]

9. Dai G, Kaazempur-Mofrad MR, Natarajan S, Zhang Y, Vaughn S, et al. Distinct endothelial phenotypes evoked by arterial waveforms derived from atherosclerosis-susceptible and -resistant regions of human vasculature. Proc. Natl. Acad. Sci. USA. 2004; 101:14871-14876. [PubMed: 15466704]

10. Fukushima T, Karino T, Goldsmith HL. Disturbances of flow through transparent dog aortic arch. Heart Vessels. 1985; 1:24-28. [PubMed: 4093352]

11. Galbraith CG, Skalak R, Chien S. Shear stress induces spatial reorganization of the endothelial cell cytoskeleton. Cell Motil. Cytoskel. 1998; 40:317-330.

12. Glagov S, Zarins C, Giddens DP, Ku DN. Hemodynamics and atherosclerosis. Insights and perspectives gained from studies of human arteries. Arch. Pathol. Lab. Med. 1988; 112:10181031. [PubMed: 3052352]

13. Goldsmith HL, Karino T. Interactions of human blood cells with the vascular endothelium. Ann. N. Y. Acad. Sci. 1987; 516:468-483. [PubMed: 3439742]

14. Hsiai TK, Cho SK, Wong PK, Ing M, Salazar A, et al. Monocyte recruitment to endothelial cells in response to oscillatory shear stress. FASEB J. 2003; 17:1648-1657. [PubMed: 12958171]

15. Huang AL, Jan KM, Chien S. Role of intercellular junctions in the passage of horseradish peroxidase across aortic endothelium. Lab. Invest. 1992; 67:201-209. [PubMed: 1501446]

16. Karino T, Goldsmith HL. Disturbed flow in models of branching vessels. Trans. Am. Soc. Art. Int. Org. 1980; 26:500-506.

17. Karino T, Goldsmith HL, Motomiya M, Mabuchi S, Sohara Y. Flow patterns in vessels of simple and complex geometries. Ann. N. Y. Acad. Sci. 1987; 516:422-441. [PubMed: 3439740]

18. Kuo CT, Veselits ML, Leiden JM. Lklf and fasl expression: corrections and clarification. Science. 1997; 278:788-789. [PubMed: 9381186] 
19. Kwak BR, Mulhaupt F, Veillard N, Gros DB, Mach F. Altered pattern of vascular connexin expression in atherosclerotic plaques. Arterioscler. Thromb. Vasc. Biol. 2002; 22:225-230. [PubMed: 11834520]

20. Li YS, Haga JH, Chien S. Molecular basis of the effects of shear stress on vascular endothelial cells. J. Biomech. 2005; 38:1949-1971. [PubMed: 16084198]

21. Li YS, Shyy JY, Li S, Lee J, Su B, et al. The Ras-JNK pathway is involved in shear-induced gene expression. Mol. Cell. Biol. 1996; 16:5947-5954. [PubMed: 8887624]

22. Lin K, Hsu PP, Chen BP, Yuan S, Usami S, et al. Molecular mechanism of endothelial growth arrest by laminar shear stress. Proc. Natl. Acad. Sci. USA. 2000; 97:9385-9389. [PubMed: 10920209]

23. Lin SJ, Jan KM, Chien S. Temporal and spatial changes in macromolecular uptake in rat thoracic aorta and relation to [3h]thymidine uptake. Atherosclerosis. 1990; 85:229-238. [PubMed: 2102086]

24. Liu Y, Chen BP, Lu M, Zhu Y, Stemerman MB, et al. Shear stress activation of SREBP1 in endothelial cells is mediated by integrins. Arterioscler. Thromb. Vasc. Biol. 2002; 22:76-81. [PubMed: 11788464]

25. Malinauskas RA, Herrmann RA, Truskey GA. The distribution of intimal white blood cells in the normal rabbit aorta. Atherosclerosis. 1995; 115:147-163. [PubMed: 7661874]

26. Masuda H, Shozawa T, Hosoda S, Kanda M, Kamiya A. Cytoplasmic microfilaments in endothelial cells of flow loaded canine carotid arteries. Heart Vessels. 1985; 1:65-69. [PubMed: 3831013]

27. Miao H, Hu YL, Shiu YT, Yuan S, Zhao Y, et al. Effects of flow patterns on the localization and expression of VE-cadherin at vascular endothelial cell junctions: in vivo and in vitro investigations. J. Vasc. Res. 2005; 42:77-89. [PubMed: 15637443]

28. Nerem RM. Hemodynamics and the vascular endothelium. J. Biomech. Eng. 1993; 115:510-514. [PubMed: 8302033]

29. Schwenke DC, Carew TE. Quantification in vivo of increased LDL content and rate of LDL degradation in normal rabbit aorta occurring at sites susceptible to early atherosclerotic lesions. Circ. Res. 1988; 62:699-710. [PubMed: 3349573]

30. Shyy JY, Lin MC, Han J, Lu Y, Petrime M, Chien S. The cis-acting phorbol ester "12-otetradecanoylphorbol 13-acetate"-responsive element is involved in shear stress-induced monocyte chemotactic protein 1 gene expression. Proc. Natl. Acad. Sci. USA. 1995; 92:8069-8073. [PubMed: 7644539]

31. Texon, M. Hemodynamic Basis of Atherosclerosis: With Critique of the Cholesterol-heart Disease Hypothesis. 2nd ed.. New York: Begell House; 1995.

32. Thoumine O, Nerem RM, Girard PR. Oscillatory shear stress and hydrostatic pressure modulate cell-matrix attachment proteins in cultured endothelial cells. In Vitro Cell Dev. Biol. Anim. 1995; 31:45-54. [PubMed: 7704333]

33. Truskey GA, Roberts WL, Herrmann RA, Malinauskas RA. Measurement of endothelial permeability to 125 i-low density lipoproteins in rabbit arteries by use of en face preparations. Circ. Res. 1992; 71:883-897. [PubMed: 1516161]

34. Tzima E, Irani-Tehrani M, Kiosses WB, Dejana E, Schultz DA, et al. A mechanosensory complex that mediates the endothelial cell response to fluid shear stress. Nature. 2005; 437:426-431. [PubMed: 16163360]

35. Walpola PL, Gotlieb AI, Langille BL. Monocyte adhesion and changes in endothelial cell number, morphology, and f-actin distribution elicited by low shear stress in vivo . Am. J. Pathol. 1993; 142:1392-1400. [PubMed: 8494043]

36. Wang N, Miao H, Li YS, Zhang P, Haga JH, et al. Shear stress regulation of Krüppel-like factor 2 expression is flow pattern-specific. Biochem. Biophys. Res. Commun. 2006; 341:1244-1251. [PubMed: 16466697]

37. Weinbaum S, Tzeghai G, Ganatos P, Pfeffer R, Chien S. Effect of cell turnover and leaky junctions on arterial macromolecular transport. Am. J. Physiol. 1985; 248:H945-H960. [PubMed: 4003572]

38. White GE, Gimbrone MA Jr, Fujiwara K. Factors influencing the expression of stress fibers in vascular endothelial cells in situ . J. Cell Biol. 1983; 97:416-424. [PubMed: 6684121] 
39. Wong AJ, Pollard TD, Herman IM. Actin filament stress fibers in vascular endothelial cells in vivo. Science. 1983; 219:867-869. [PubMed: 6681677]

40. Zhao Y, Chen BP, Miao H, Yuan S, Li YS, et al. Improved significance test for DNA microarray data: temporal effects of shear stress on endothelial genes. Physiol. Genomics. 2002; 12:1-11. [PubMed: 12399447] 


\section{(a) Rectangular Flow Chamber}

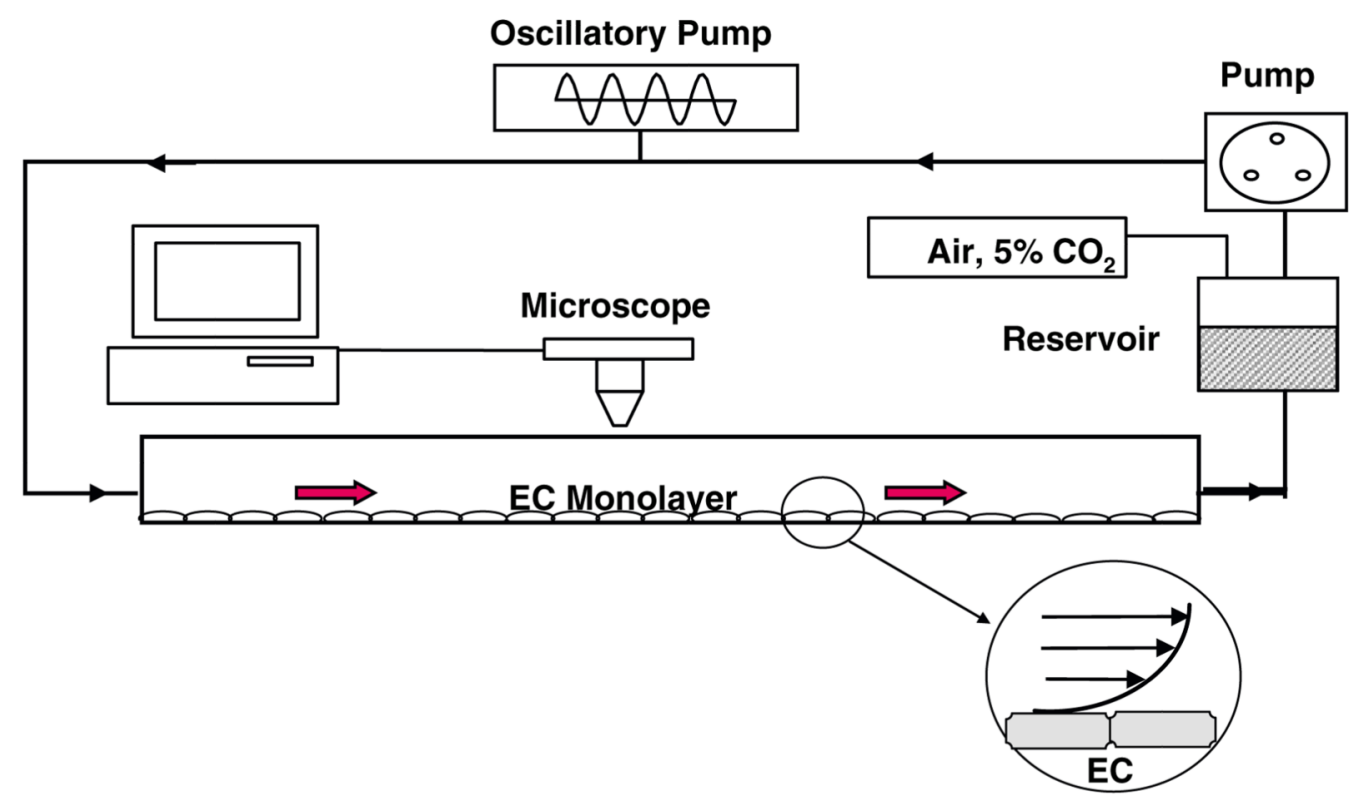

(b)

Step Flow Chamber

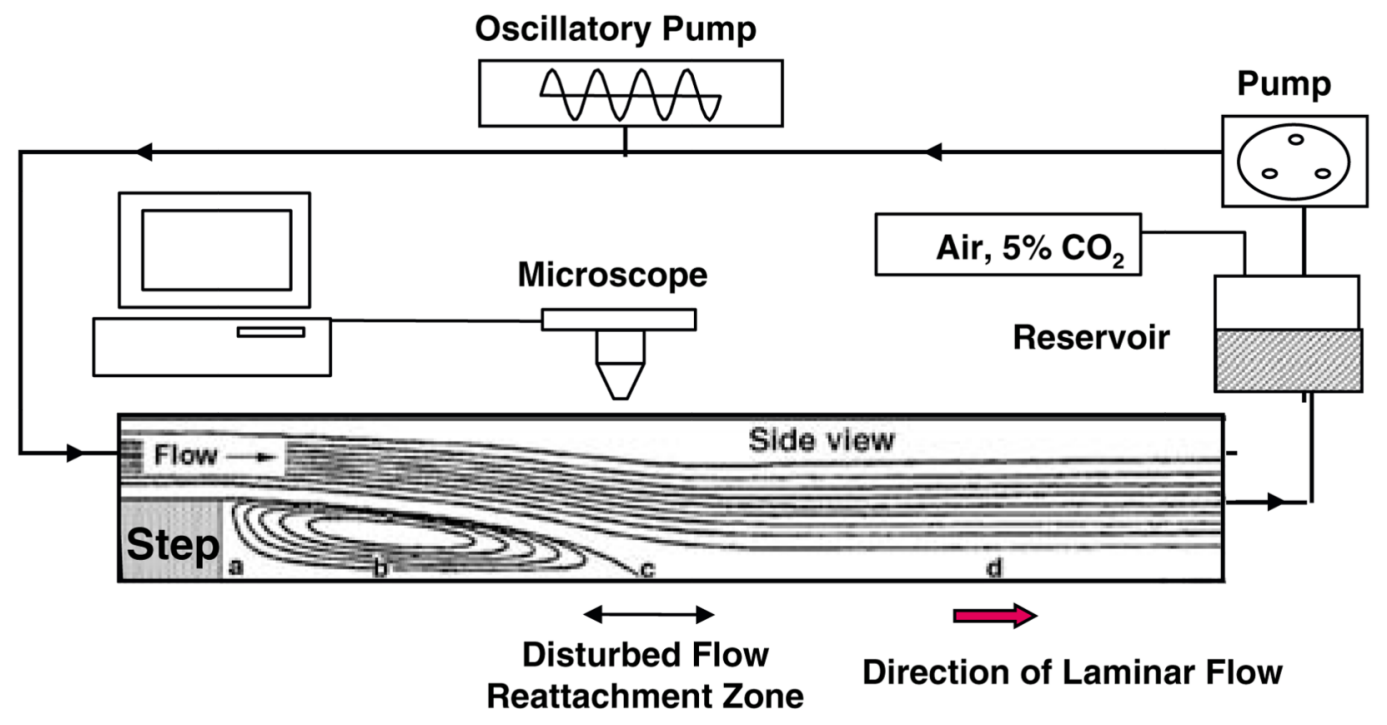

FIGURE 1.

Side views of rectangular flow chamber (a) and step flow chamber (b). In both bases, the shear flow can be either steady without oscillations or superimposed with sinusoidal oscillation by using the oscillatory pump. The EC monolayer is shown only in (a); the inset in (a) shows an enlarged view of two ECs and the velocity gradient due to the applied shear stress. In (b), the flow direction in zone b is opposite to the incoming flow and to that in zone $\mathrm{d}$, where forward flow is re-established. Zone $\mathrm{c}$ is the reattachment zone where the flow fluctuates between the forward and backward directions, with a low shear stress but large shear stress gradient; this is the area of disturbed flow. From Chien.4 


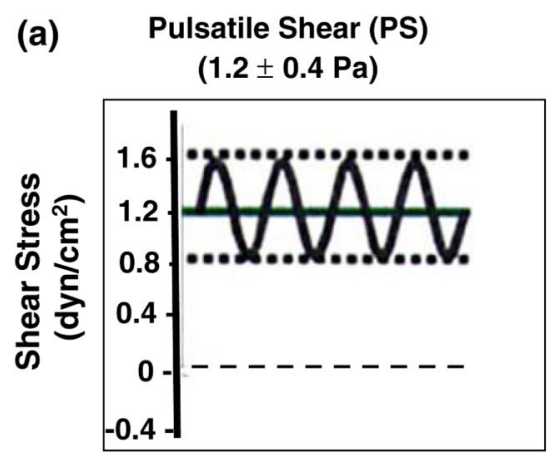

(b) Reciprocating Shear (RS)
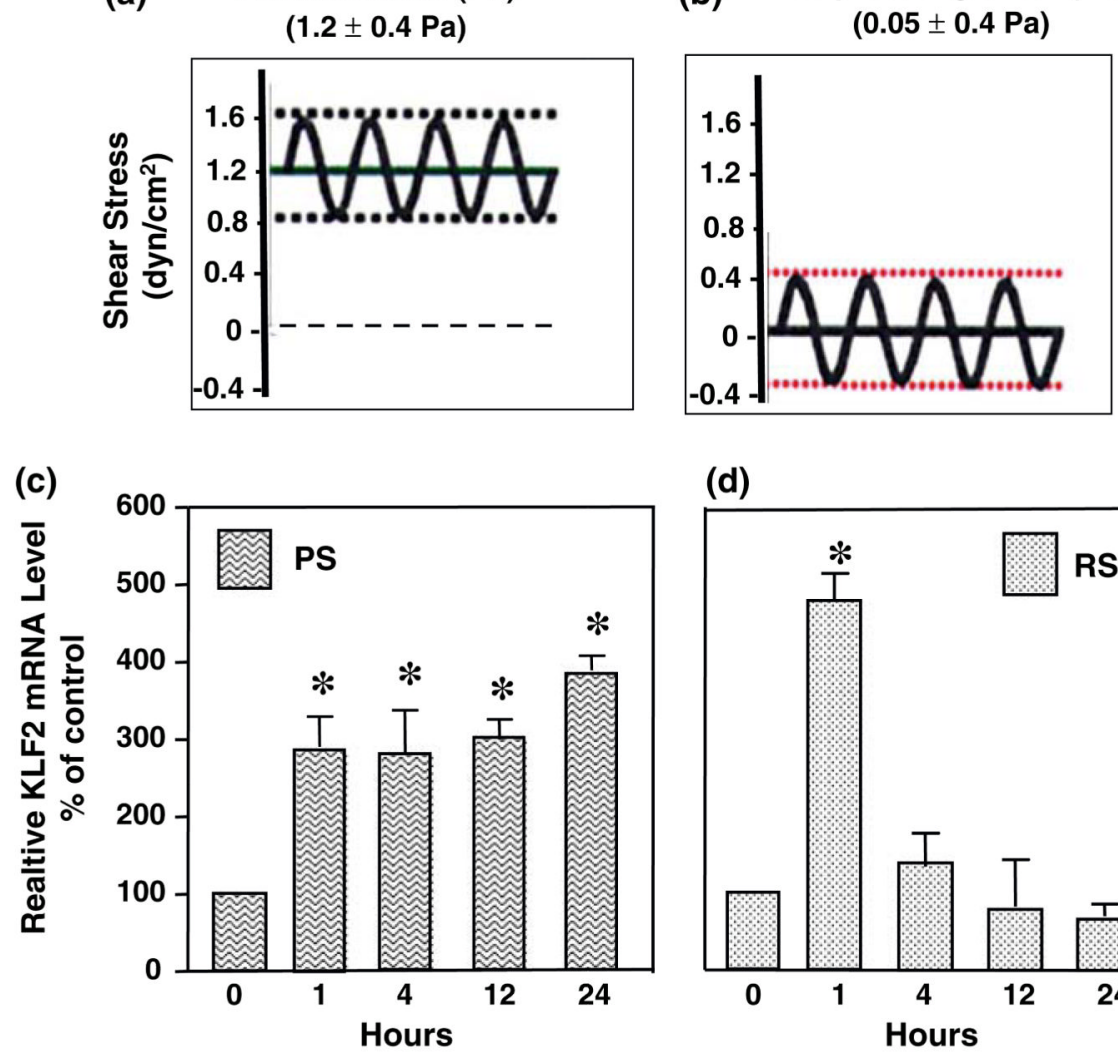

(d)

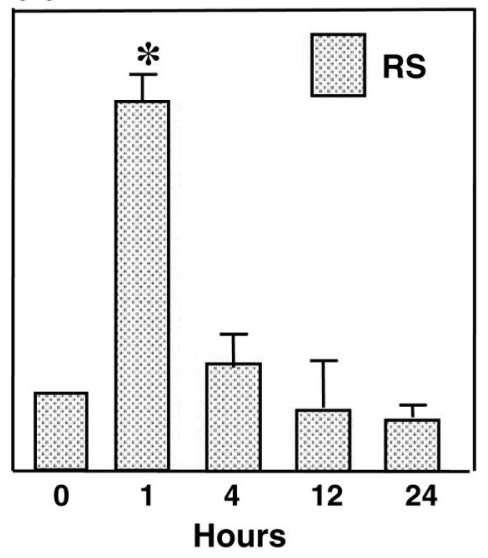

FIGURE 2.

Flow-pattern-specific regulation of KLF2 gene expression in human umbilical vein endothelial cells. (a) Pulsatile flow with a sinusoidal oscillatory shear stress of $1.2 \pm 0.4 \mathrm{~Pa}$. (b) Reciprocating shear stress with a very low mean shear stress of $0.05 \mathrm{~Pa}$ (to maintain nutrient delivery) and a sinusoidal oscillation of $\pm 0.4 \mathrm{~Pa}$. (c) KLF2 mRNA levels of confluent ECs subjected to pulsatile shear at $1.2 \pm 0.4 \mathrm{~Pa}$ (PS) for $0,1,4,12$, and $24 \mathrm{~h}$. Note the sustain elevation of KLF-2 over the $24 \mathrm{~h}$ of study. (d) KLF2 mRNA levels of confluent ECs subjected to reciprocating shear at $0.05 \pm 0.4 \mathrm{~Pa}$ (RS) for 0, 1, 4, 12, and $24 \mathrm{~h}$. Note the transient elevation of KLF-2 followed by a decline to a level below control at $24 \mathrm{~h}$. Based on Wang et al.36 


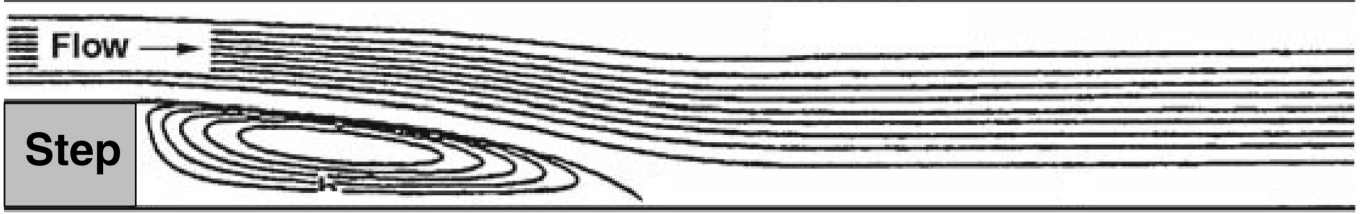

\section{Disturbed Flow Reattachment Zone}

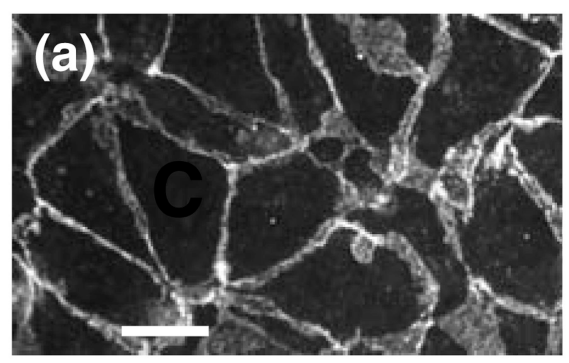

Static Control

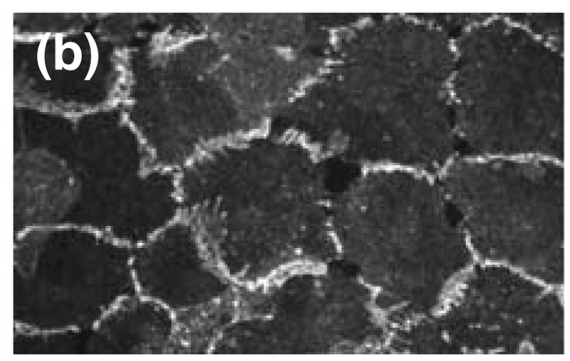

Disturbed Flow
Direction of laminar Flow

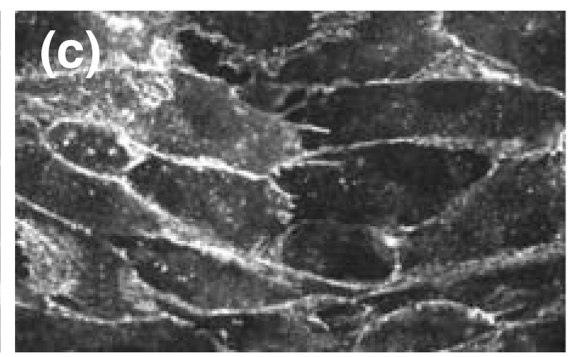

Laminar Flow

FIGURE 3.

VE-cadherin staining in endothelial cells exposed to disturbed and laminar flows in vitro. Confluent monolayers of BAECs were kept as controls or subjected to disturbed and laminar flows in a step flow chamber. Staining of VE-cadherin was observed by confocal microscopy. VE-cadherin staining was continuously distributed around the entire periphery of cells under static condition (a). After a 24-h exposure to the disturbed flow, the VEcadherin distribution became discontinuous (b), whereas 24-h exposure to laminar flow resulted in a continuous distribution of VE-cadherin (c), as in the static condition. Images shown here are representative results from 3 separate experiments. Bar in a $=30 \mu \mathrm{m}$. From Miao et al. 27 


\section{(a) Step Flow Channel}

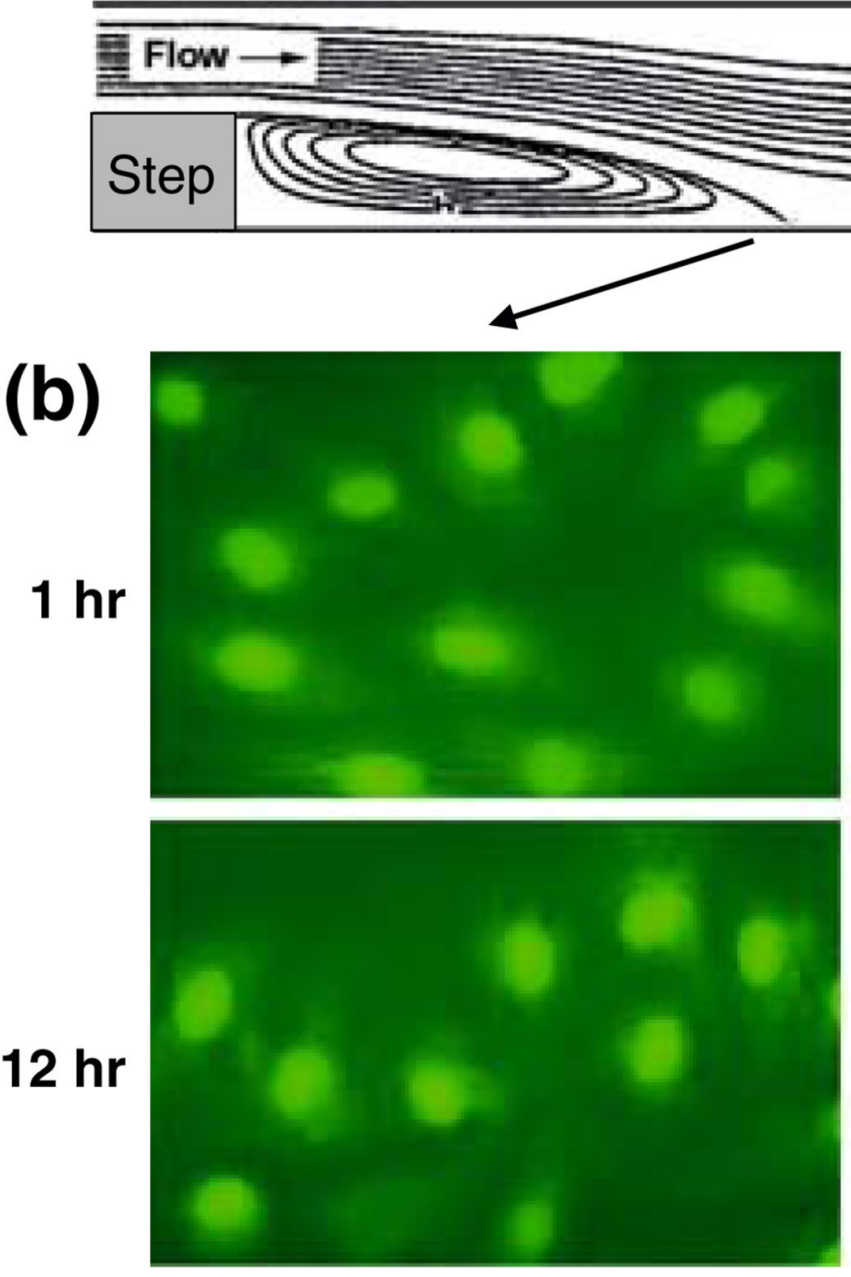

Disturbed Flow

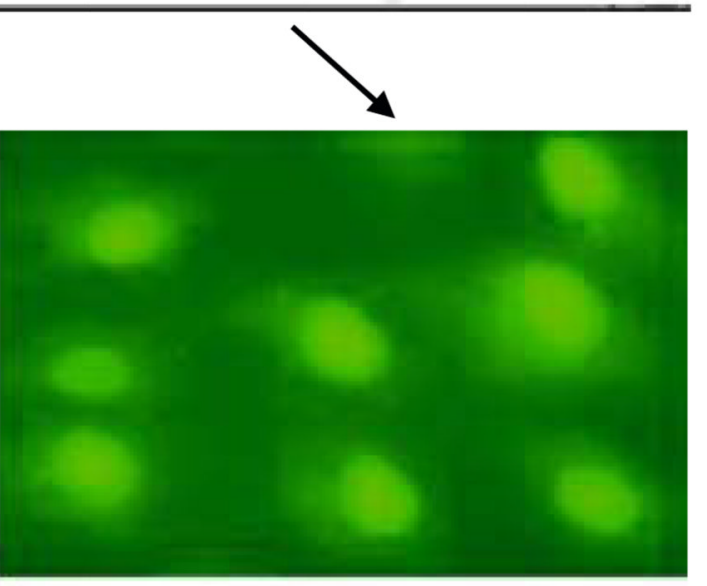

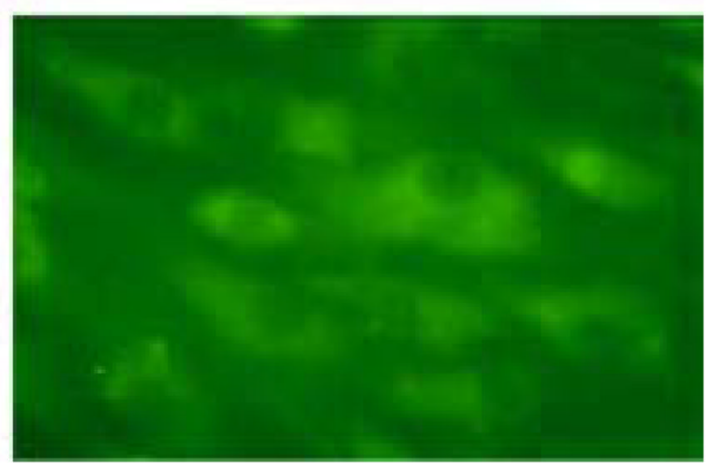

Laminar Flow

\section{FIGURE 4.}

Effects of flow pattern on the activation of SREBP1 in a step flow chamber. (a) Shows side view of the step flow chamber in which confluent cultured BAECs are subjected to different flow patterns. After shearing for 1 or $12 \mathrm{~h}$, the cells were fixed and immunostained for SREBP1. While disturbed flow induced a sustained activation of SREBP1, as indicated by its translocation into the nuclei (b, Lower left), laminar flow activated SREBP1 in a transient manner, as evidenced by the lack of nuclear staining of SREBP1 at $12 \mathrm{~h}$ (b, lower right). Modified from Liu et al. 24 


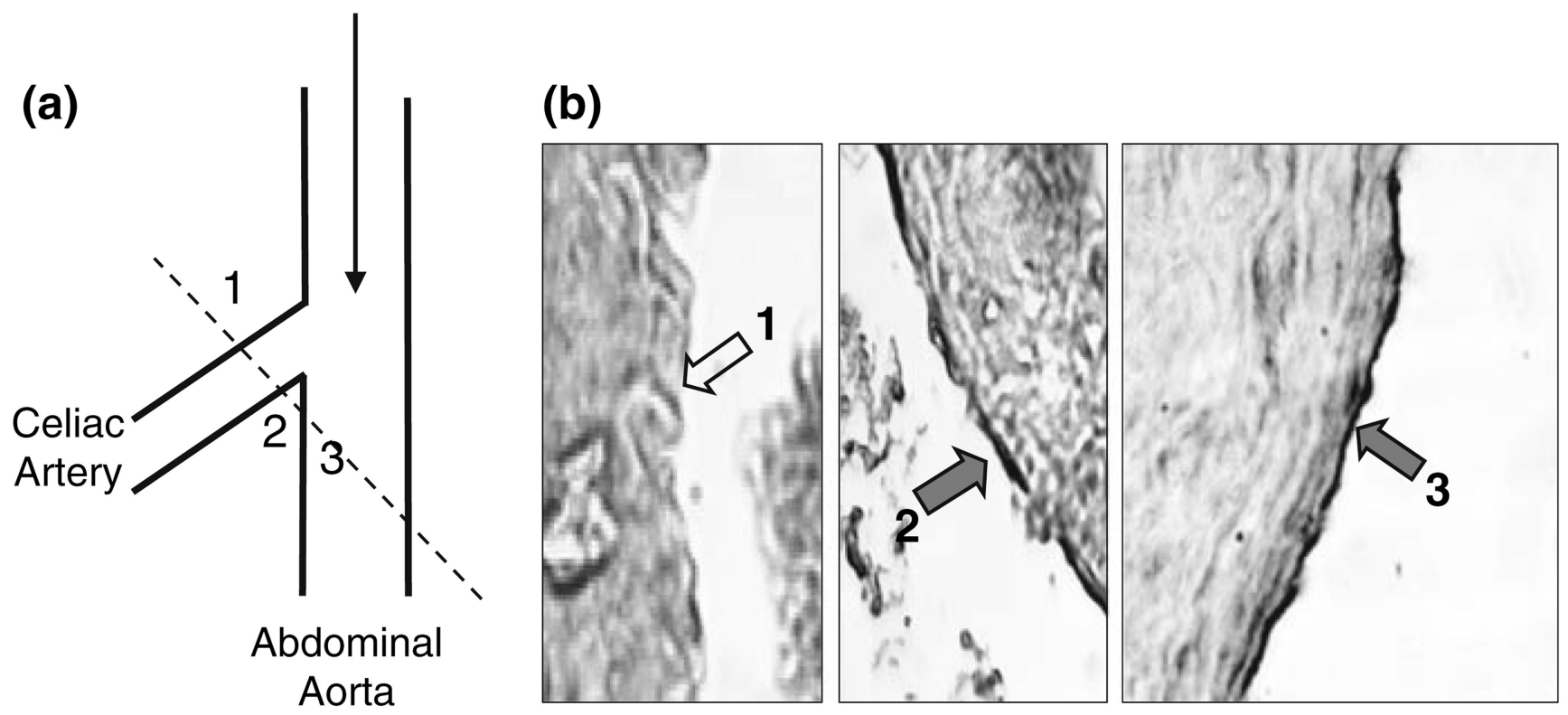

FIGURE 5.

KLF2 Expression in ECs at abdominal-celiac branch point. (a) Drawing to show the branch point of celiac artery off the abdominal aorta. The broken line shows the section through which the samples were taken for immunohistochemical examination of KLF2 expression in b. (b) Cross section of the branch area with areas 1,2, and 3 shown in the three pictures. The expression of KLF2 was high and continuous on ECs of the abdominal aorta (labeled 3) and the medial aspect (labeled 2) of the celiac branch, but virtually absent on the lateral aspect (labeled 1) of the branch. These results are representative of three independent experiments. In (b), the short filled arrows ( 2 and 3 ) indicate the positive staining for KLF2 protein, and the open arrow (1) shows the absence of KLF2 protein in the endothelium. Modified from Wang et al. 36 


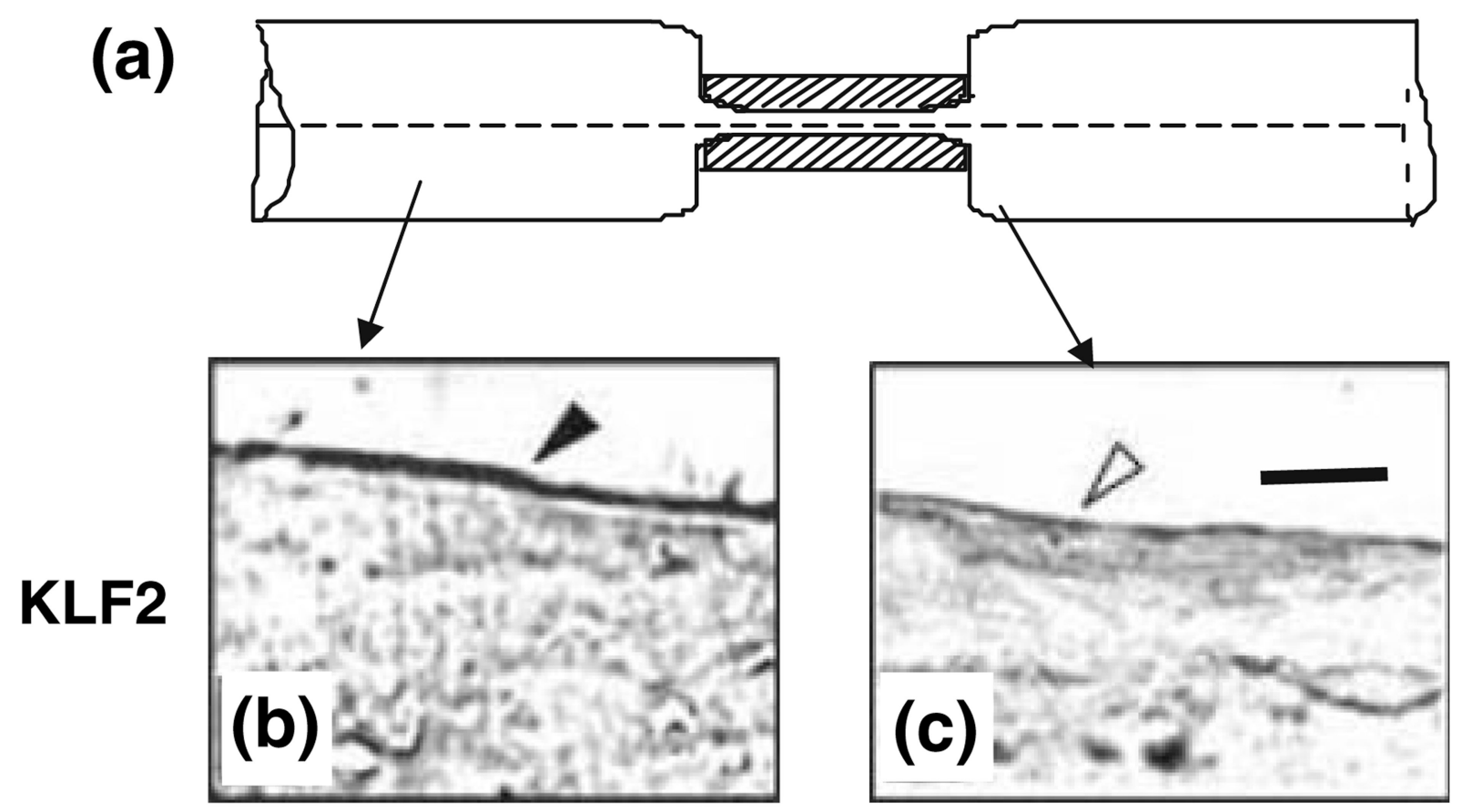

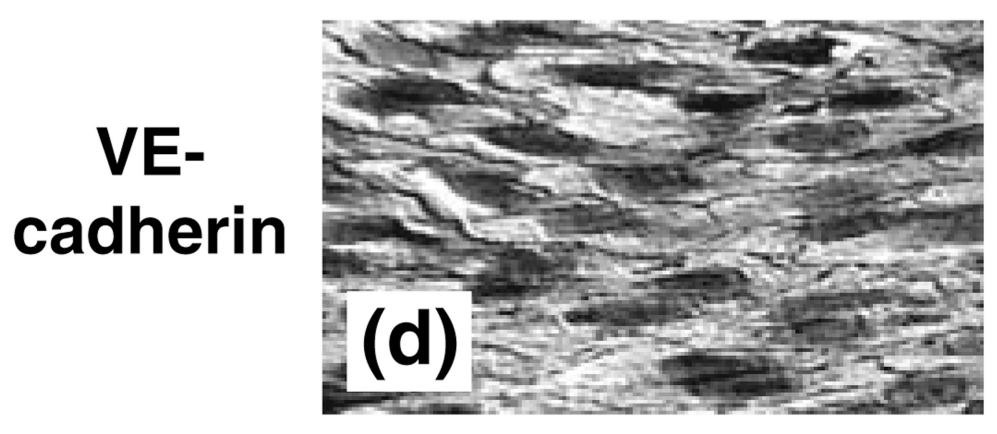

Laminar Flow

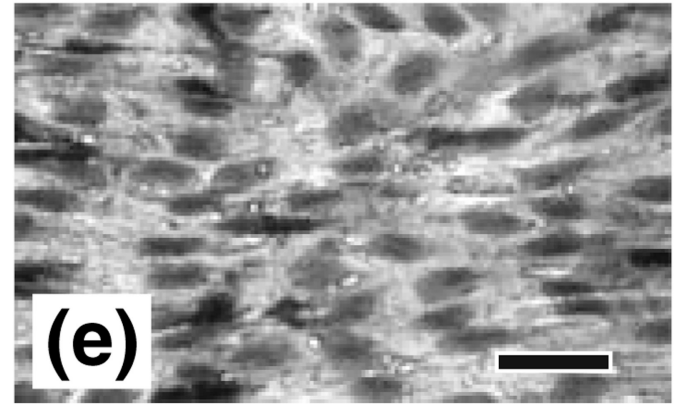

Disturbed Flow

FIGURE 6.

(a) Side view of in the rat abdominal aorta with a local stenosis created by using a U-shaped titanium clip, with the aim of studying the effects of flow patterns on KLF2 expression and VE-cadherin distribution in vivo. Four weeks after the creation of stenosis, the vessel was harvested, fixed, embedded, sectioned longitudinally, and subjected to immunohistochemistry staining with anti-KLF2 (b and c) or anti-VE-cadherin ( $\mathrm{d}$ and e). Blood flow is from left to right, as indicated by the arrow. (b) Endothelium in a laminar flow region $5 \mathrm{~mm}$ upstream of the stenosis site shows strong KLF2 staining (closed arrowhead). The downstream laminar flow region (not shown) has the same strong staining. (c) the poststenotic site distal to the clip, which has disturbed flow pattern, shows a lack of KLF2 staining (open arrowhead). Bar in $\mathrm{c}=10 \mu \mathrm{m}$. (d) In the laminar flow region $5 \mathrm{~mm}$ upstream (as well as $5 \mathrm{~mm}$ downstream) to the clip site, VE-cadherin is highly expressed at endothelial cell borders. (e) No detectable VE-cadherin is found at cell borders in the disturbed flow region immediately downstream to the constriction. Bar in e $=30 \mu \mathrm{m}$. Based on Wang et al. 36 and Miao et al. 27 

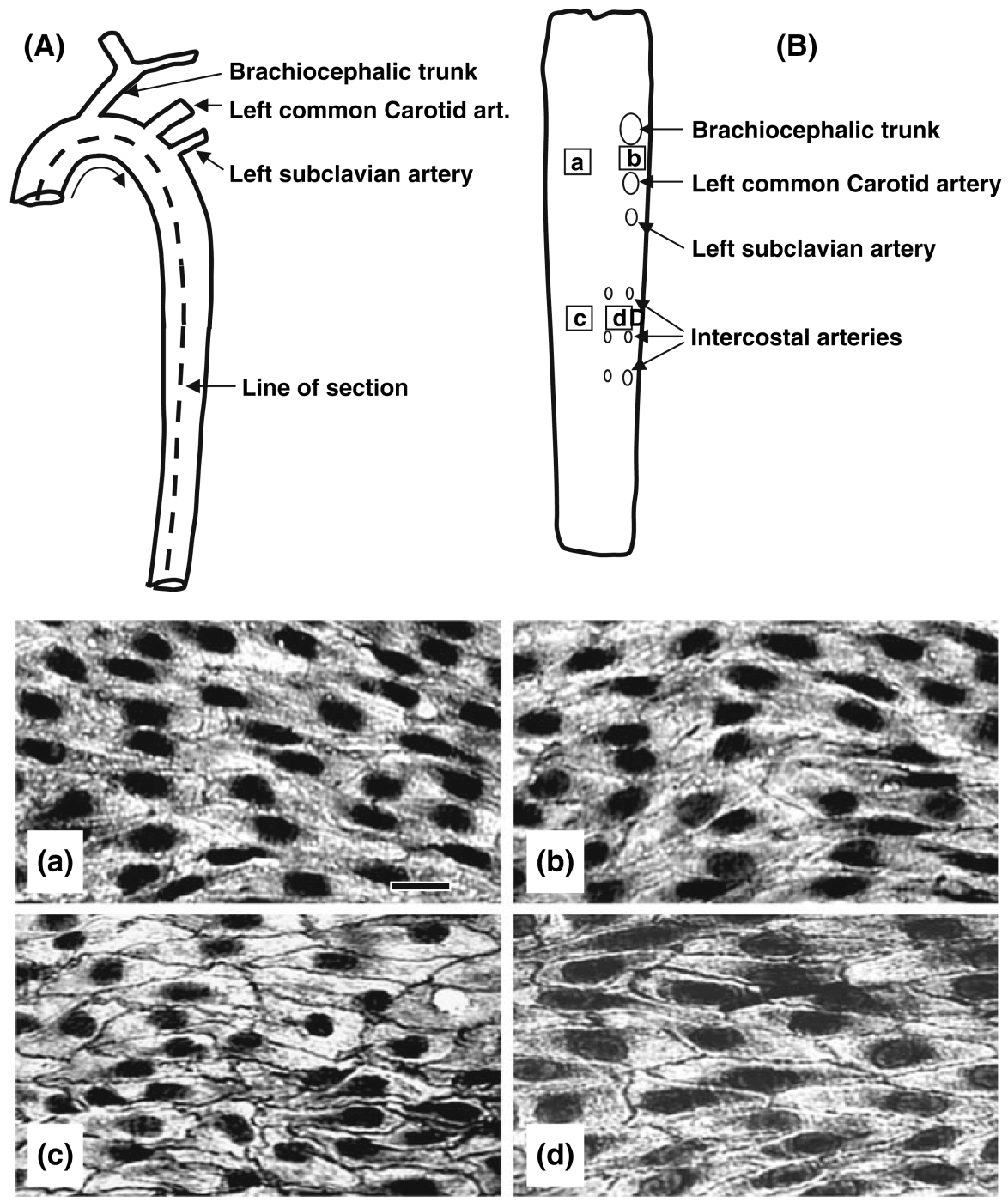

\section{FIGURE 7}

Schematic diagrams of the rat thoracic aorta, showing its overall anatomy (A) and its luminal surface after having been cut open and pinned flat (B), the labels a, b, c and d in B indicate the areas from which the four pictures (a, b, c, and d) for VE-cadherin staining were taken by en face staining with an anti-VE-cadherin antibody. The VE-cadherin staining at endothelial cell borders was weak and discontinuous in the aortic arch (a: inferior and b: superior aspects), where disturbed flow dominates, and was much stronger and continuous in the descending thoracic aorta with laminar flow (c: dorsal and d: ventral aspects). Bar in a $=30 \mu \mathrm{m}$. Based on Miao et al. 27 
TABLE 1

Comparison of flow patterns, cellular events and atherogenecity between straight part of the aorta and its branch points.

\begin{tabular}{lll}
\hline Arterial vessels & Straight part & Branch points \\
\hline Flow pattern & Laminar & Disturbed \\
Net forward flow & Large & Small \\
$\begin{array}{l}\text { Stress fiber alignment } \\
\text { Monocyte adhesion }\end{array}$ & Along long axis & Not aligned \\
$\begin{array}{l}\text { EC turnover \& LDL } \\
\text { permeability }\end{array}$ & Low & High \\
$\begin{array}{l}\text { Inflammatory genes } \\
\text { Genes to raise intracellular }\end{array}$ & Down-regulated & Up-regulated \\
$\begin{array}{l}\text { lipids } \\
\text { Effect on atherogenesis }\end{array}$ & Anti-atherogenic & Pro-atherogenic \\
\hline
\end{tabular}

